Check for updates

Cite this: RSC Adv., 2018, 8, 34483

\title{
Daphnetin inhibits proliferation and glycolysis in colorectal cancer cells by regulating the PI3K/Akt signaling pathway
}

\author{
Zhikuan He, $\uparrow^{\mathrm{a}}$ Wenxing Dong, $\hat{\dagger}^{\mathrm{b}}$ Kunhou Yao, ${ }^{\mathrm{a}}$ Changjiang Qin ${ }^{\mathrm{a}}$ \\ and Baomin Duan (D) *b
}

Daphnetin (7,8-dihydroxycoumarin), a natural coumarin compound, has shown antitumor and energy metabolism regulatory activities. However, the effects of daphnetin on cell proliferation, migration, and glucose metabolism in colorectal cancer (CRC) cells remains unknown. In this study, the effects of daphnetin on CRC cell proliferation, migration, and glucose metabolism have been examined. The results showed that daphnetin inhibited the proliferation, migration, and invasion of CRC cells, and induced CRC cell apoptosis. Furthermore, daphnetin suppressed intracellular glucose and lactate production, and downregulated the expression of hexokinase 2 (HK2) and glucose transporter 1 (GLUT1) in CRC cells. Furthermore, daphnetin prevented activation of the PI3K/Akt pathway in CRC cells. These findings demonstrated that daphnetin inhibited the proliferation, migration and glucose metabolism in CRC cells by suppressing the PI3K/Akt signaling pathway. Therefore, daphnetin has potential as a novel anticancer agent for CRC treatment.

Received 29th June 2018

Accepted 21st September 2018

DOI: $10.1039 / c 8 r a 05583 a$

rsc.li/rsc-advances

\section{Introduction}

Colorectal cancer (CRC) is a leading cause of cancer-related death worldwide. ${ }^{1}$ Although much progress has been made in CRC diagnosis and treatment in the last few decades, most patients are first diagnosed at an advanced stage and the fiveyear survival of patients with distant metastasis is less than $10 \% .^{2-4}$ Therefore, exploration of the molecular mechanism of CRC carcinogenesis is urgently needed.

Aerobic glycolysis, a hallmark of various tumors, is characterized by increased glucose uptake, enhanced glycolysis, dysregulated mitochondrial oxidative phosphorylation, and accumulated lactate production. ${ }^{5}$ Tumor cells gain a substantial amount of their energy from aerobic glycolysis. ${ }^{6}$ Therefore, targeting aerobic glycolysis in cancer cells is a good method for preventing CRC progression.

Daphnetin (7,8-dihydroxycoumarin) is a natural coumarin compound that has been reported to possess various pharmacological activities, such as anti-inflammatory, immunosuppressive, antioxidant, neuroprotective, and antitumor effects. ${ }^{7-9}$ A recent study reported that daphnetin dose-dependently

${ }^{a}$ Department of General Surgery, Henan University Huaihe Hospital, Kaifeng 475000, Henan Province, China

${ }^{b}$ Department of Emergency, Kaifeng Central Hospital, 58 East Street, Kaifeng 475000, Henan Province, China. E-mail: baomin_duankf@126.com; Fax: +86-0371-25672905; Tel: +86-0371-25672905

$\dagger$ Zhikuan He and Wenxing Dong contributed equally to this study and should be considered co-first authors. inhibits the migration and invasion of murine osteosarcoma LM8 cells. ${ }^{10}$ Previous studies have reported that daphnetin could alter energy metabolism in several diseases. For example, daphnetin pretreatment significantly reduced the levels of lipid peroxidation markers in STZ-induced INS-1 cells. ${ }^{11}$ However, the effects of daphnetin on cell proliferation, migration, and glucose metabolism in CRC cells remain unknown. Therefore, in this study, we examined the effects of daphnetin on CRC cell proliferation, migration, and glucose metabolism.

\section{Materials and methods}

\section{Cell culture}

Human CRC cell lines (SW480 and HT-29) were obtained from the American Type Culture Collection (ATCC, Manassas, VA, USA). All cells were cultured in Dulbecco's modified Eagle's medium (DMEM; Hyclone, Logan, UT, USA) containing 10\% (v/ v) fetal bovine serum (FBS; Gibco, Rockville, MD) and 100 units per $\mathrm{mL}$ of streptomycin and penicillin (Sigma, St. Louis, MO, USA) at $37{ }^{\circ} \mathrm{C}$ in a humidified incubator with $5 \% \mathrm{CO}_{2}$.

\section{Cell proliferation assay}

Cell proliferation was measured using the 3-(4,5-methylthiozol2-yl)-2,5-diphenyltetrazolium bromide (MTT) assay. In brief, CRC cells $\left(1 \times 10^{4}\right.$ cells per well) were seeded into 96-well plates and treated with various daphnetin concentrations (25 and 50 $\mu \mathrm{M})$ for $24 \mathrm{~h}$. Then, MTT ( $20 \mu \mathrm{L}, 5 \mathrm{mg} \mathrm{mL}^{-1}$; Sigma) was added and cells were cultured for an additional $4 \mathrm{~h}$. The supernatant 
was carefully removed and dimethylsulfoxide (DMSO, $100 \mu \mathrm{L}$; Sigma) was added to lyse the cells. The absorbance (OD) was measured using a microplate reader (Bio-Rad, Hercules, CA, USA) at a wavelength of $570 \mathrm{~nm}$.

\section{Cell migration and invasion assay}

Cell migration and invasion assays were performed using transwell chamber assays. For the migration assay, CRC cells ( 1 $\times 10^{4}$ cells per well) were suspended in serum-free medium and plated on the upper chamber (BD Biosciences, Bedford, MA, USA), while DMEM with $10 \%$ FBS $(500 \mu \mathrm{L})$ was added into the lower chamber. The assays for migration and invasion were conducted for $24 \mathrm{~h}$. Cells remaining on the upper surface of the membrane were wiped off with a cotton swab. The migrated cells on the lower surface of the filter were fixed, stained, and counted under a light microscope (Olympus, Tokyo, Japan). For the invasion assay, the procedures described above were used, except that the filters were precoated with Matrigel (BD Biosciences) to form a matrix barrier.

\section{Cell apoptosis assay}

Cell apoptosis was evaluated using ELISA-based cell death detection kit (Roche Molecular Biochemicals, Mannheim, Germany) based on the principle of quantifying apoptosis-specific DNA fragmentation according to a previously described method. DNA fragmentation was measured using a microplate reader, with the absorbance recorded at $405 \mathrm{~nm}$.

\section{Western blot}

Total protein of the CRC cells was prepared using RIPA lysis buffer (Beyotime, Nantong, China). The protein concentration was detected using a Bradford protein assay kit (Bio-Rad). A total of $50 \mu \mathrm{g}$ of protein was isolated by $12 \%$ sodium dodecyl sulfate polyacrylamide gel electrophoresis (SDS-PAGE), followed by electro-blotting-mediated transfer onto polyvinylidene difluoride (PVDF) membranes (Millipore, Billerica, MA, USA). The membranes were then blocked in 5\% non-fat milk in TBST buffer (5 mM Tris-HCl, pH 7.4, 136 mM NaCl, 0.1\% Tween 20) for $1 \mathrm{~h}$ at room temperature, and then incubated with primary antibodies against bcl-2, bax, cleaved caspase-3, hexokinase 2 (HK2), glucose transporter 1 (GLUT1), p-PI3K, PI3K, p-Akt, Akt, and GAPDH (Cell Signaling, Danvers, MA, USA) overnight at $4{ }^{\circ} \mathrm{C}$. After washing three times with TBST, the membranes were incubated with the appropriate HRP-conjugated secondary antibodies (Cell Signaling) for $1 \mathrm{~h}$. Finally, the immunocomplexes were visualized by chemiluminescence using an ECL kit (Amersham Biosciences, Piscataway, NJ, USA).

\section{Glucose uptake level and lactate generation measurements}

After treatment, the glucose uptake was evaluated using a glucose assay kit (BioVision) according to the manufacturer instructions. The level of lactate in the supernatant was detected using a Lactate Colorimetric Assay Kit (Biovision Inc.), according to the manufacturer instructions.

\section{Statistical analysis}

All statistical analyses were performed using SPSS version 13.0 (SPSS Inc., USA). Data are presented as mean \pm SD. The statistical difference was analyzed using Student's $t$-test between two groups and one-way analysis of variance (ANOVA) among multiple groups. $P<0.05$ was considered to indicate a statistically significant difference.

\section{Results}

\section{Effect of daphnetin on CRC cell proliferation}

Firstly, we examined the effect of daphnetin on CRC cell proliferation using the MTT assay. As shown in Fig. 1A, compared with untreated SW480 cells, daphnetin treatment significantly repressed SW480 cell proliferation in a dose-

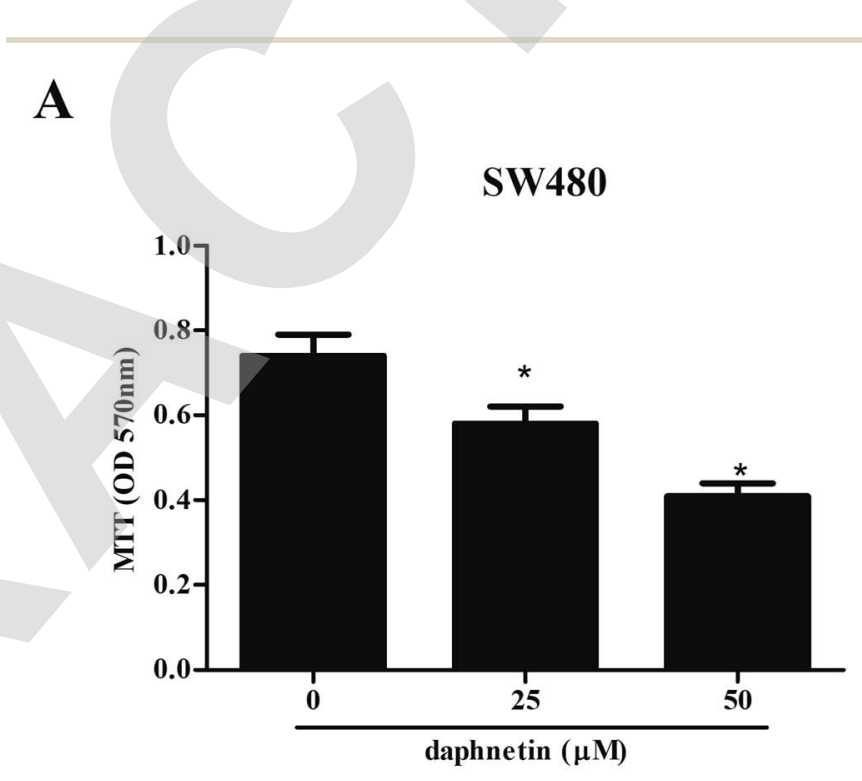

B

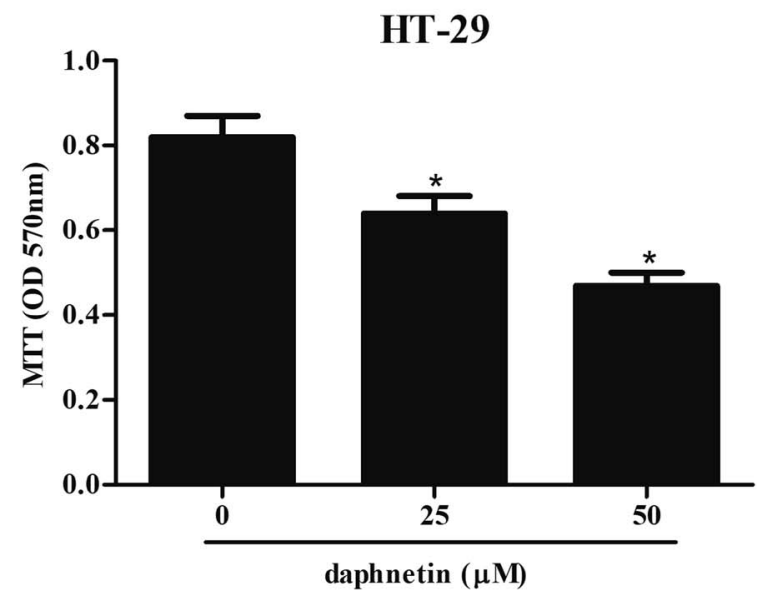

Fig. 1 Effect of daphnetin on CRC cell proliferation. CRC cells $\left(1 \times 10^{4}\right.$ cells per well) were treated with various daphnetin concentrations $(0$, 25, and $50 \mu \mathrm{M}$ ) for $24 \mathrm{~h}$. (A) Cell proliferation detected using the MTT assay in SW480 cells. (B) Cell proliferation measured using the MTT assay in HT-29 cells. ${ }^{*} p<0.05$ vs. control group. 
independent manner. Similarly, HT-29 cell proliferation was also suppressed by daphnetin (Fig. 1B).

\section{Effect of daphnetin on CRC cell migration and invasion}

We then examined the effects of daphnetin on CRC cell migration and invasion using the transwell assays. As shown in Fig. 2A, the number of migrated cells was significantly lower in SW480 cells treated with daphnetin compared with the control group. The results of the Matrigel invasion assay indicated that daphnetin treatment also decreased SW480 cell invasion
(Fig. 2B). Similarly, daphnetin markedly reduced the migratory and invasive capabilities of HT-29 cells compared with the control group (Fig. 2C and D).

\section{Effect of daphnetin on CRC cell apoptosis}

We next investigated the effect of daphnetin on cell apoptosis, with the results of the cell death detection ELISA kit indicating that daphnetin treatment significantly induced cell apoptosis both in SW480 and HT-29 cells compared with the control groups (Fig. 3A and B). Furthermore, expressions of apoptosis-
A
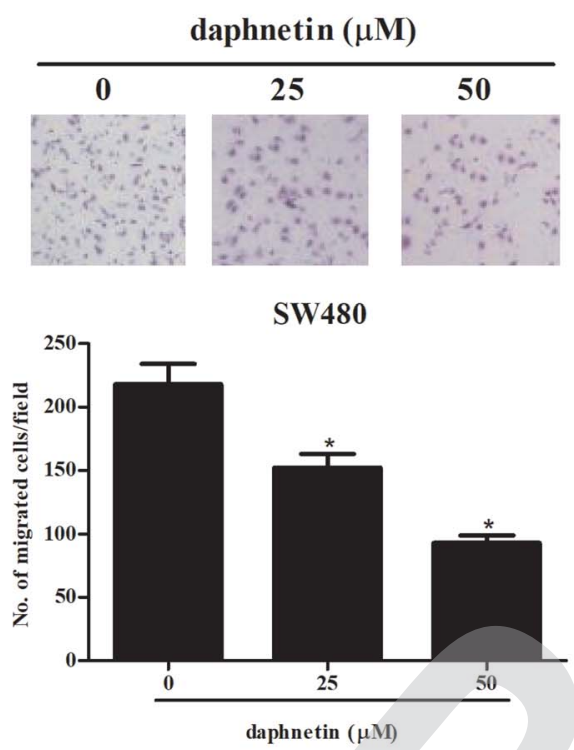

C
B
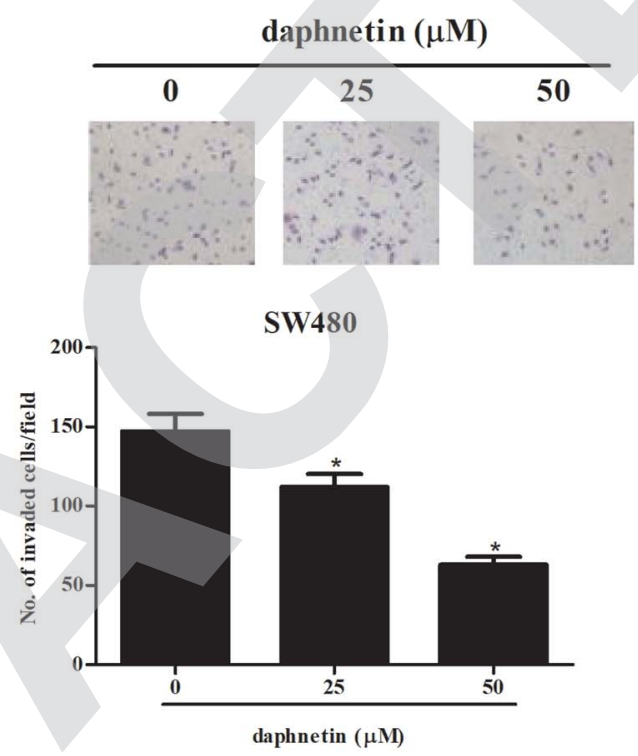

D
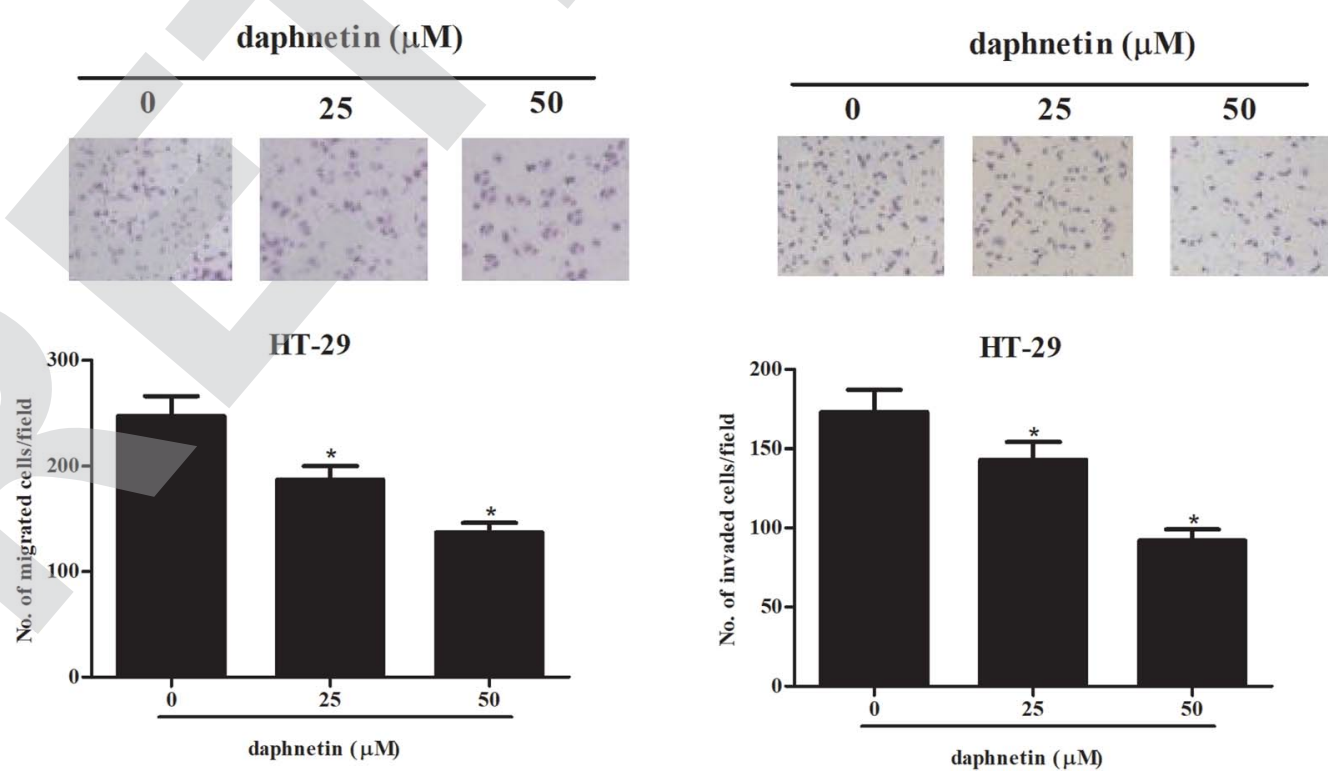

Fig. 2 Effect of daphnetin on CRC cell migration and invasion. CRC cells $\left(1 \times 10^{4}\right.$ cells per well) were treated with various daphnetin concentrations $(0,25$, and $50 \mu \mathrm{M})$ for $24 \mathrm{~h}$. (A and C) Cell migration detected using the transwell migration assay in (A) SW480 and (C) HT-29 cells. (B and D) Cell invasion measured using the Matrigel invasion assay in (B) SW480 and (D) HT-29 cells. * $p<0.05$ vs. control group. 
A

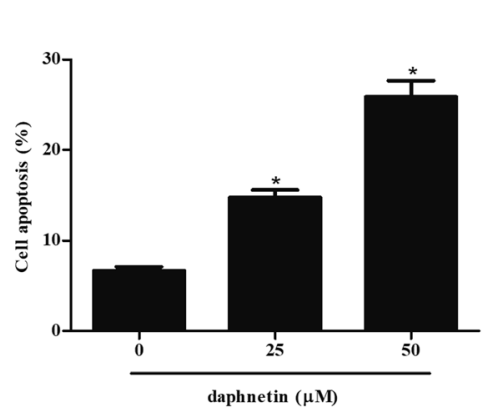

C

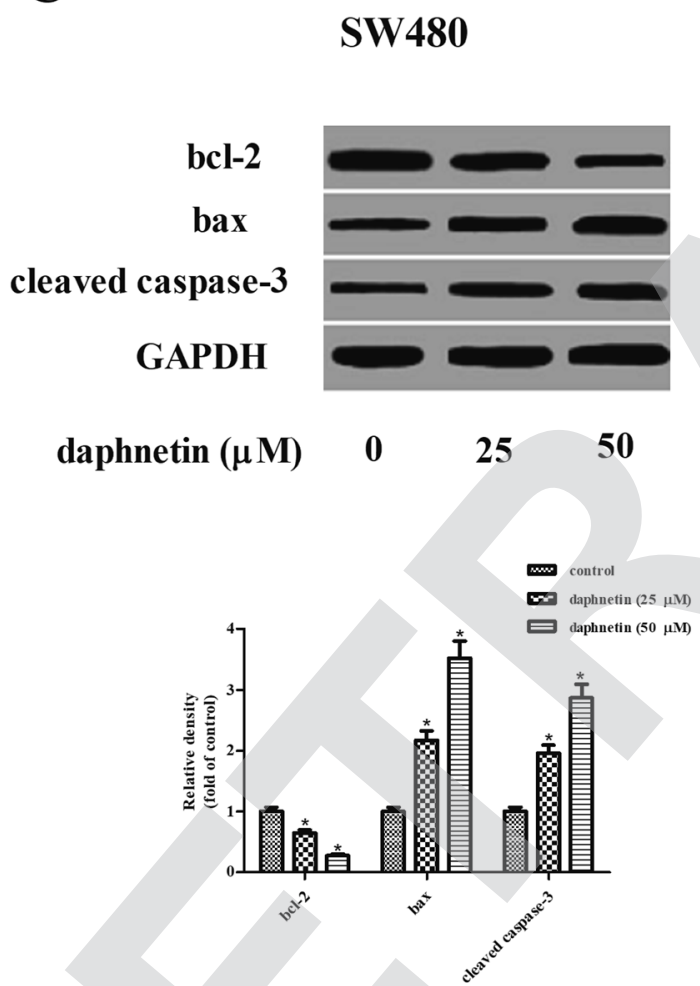

\section{SW480}

B

D

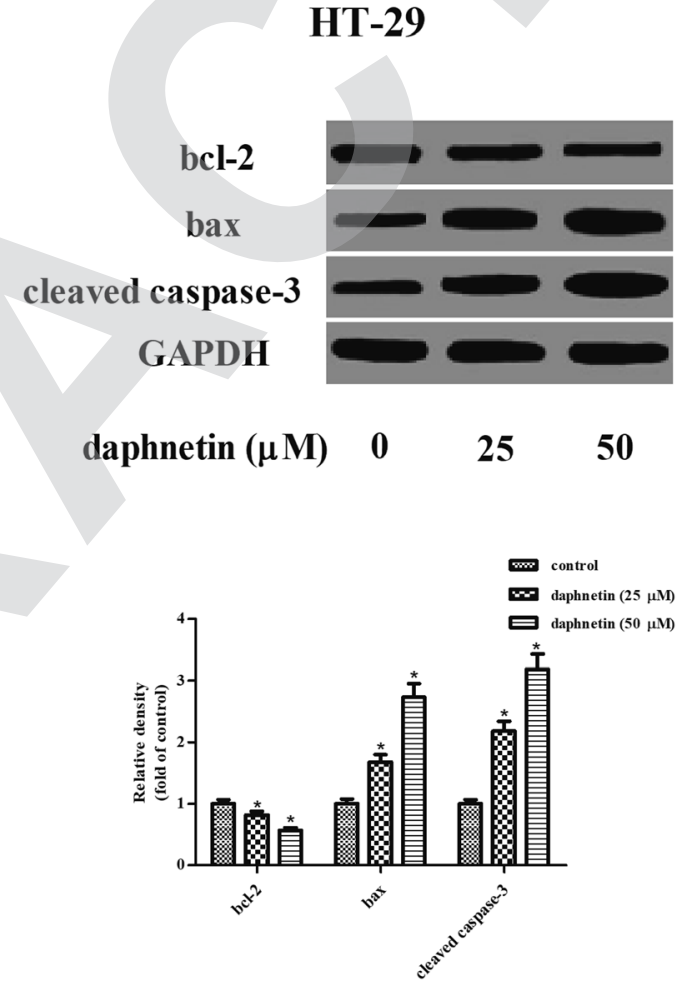

Fig. 3 Effect of daphnetin on CRC cell apoptosis. CRC cells $\left(1 \times 10^{4}\right.$ cells per well) were treated with various daphnetin concentrations $(0,25$, and $50 \mu \mathrm{M}$ ) for $24 \mathrm{~h}$. (A and B) Cell apoptosis evaluated using cell death detection ELISA kit. (C and D) Protein expression levels of bcl-2, bax, and cleaved caspase- 3 detected using western blot in SW480 and HT-29 cells. ${ }^{*} p<0.05$ vs. control group.

related proteins bcl-2, bax, and cleaved caspase-3 were detected using western blot analysis. As shown in Fig. 3C and D, daphnetin treatment downregulated the expression of bcl-2 and upregulated the expression of bax and cleaved caspase- 3 in SW480 and HT-29 cells, respectively.

\section{Effect of daphnetin on glycolysis in CRC cells}

We next examined the effects of daphnetin on glucose uptake and lactate production in CRC cells. As shown in Fig. 4A and B, intracellular glucose in SW480 and HT-29 cells was gradually suppressed by daphnetin treatment. Similarly, daphnetin treatment significantly reduced lactate production in SW480 and HT-29 cells (Fig. 4C and D). Furthermore, the protein expression levels of HK2 and GLUT1 were also examined. The results of western blot analysis indicated that daphnetin treatment significantly suppressed HK2 and GLUT1 expression in SW480 and HT-29 cells, respectively (Fig. 4E and F).

\section{Effect of daphnetin on PI3K/Akt pathway in CRC cells}

As the PI3K/Akt signaling pathway can regulate cell proliferation, migration, and glucose metabolism in several types of tumor, we attempted to investigate the effect of daphnetin on 
A

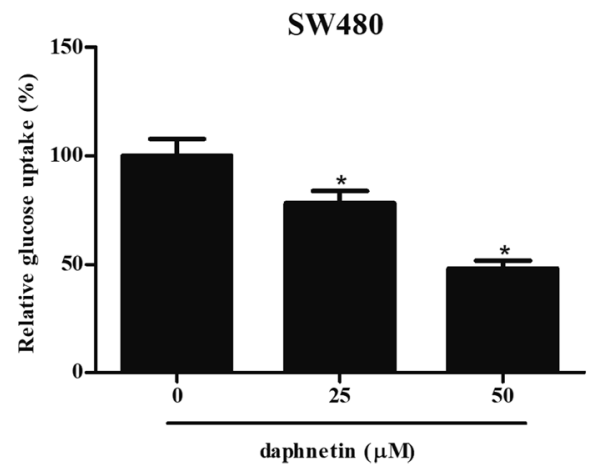

C

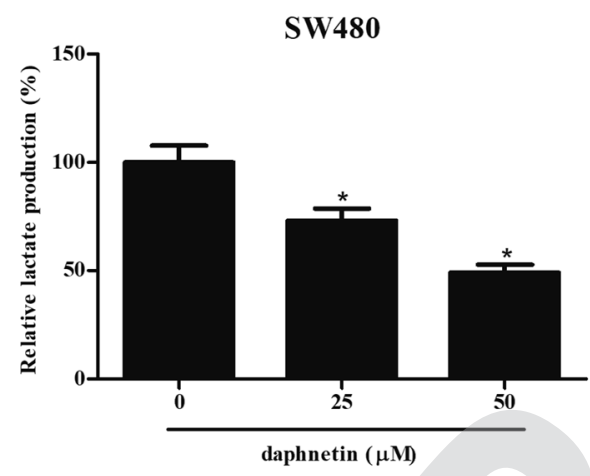

$\mathbf{E}$
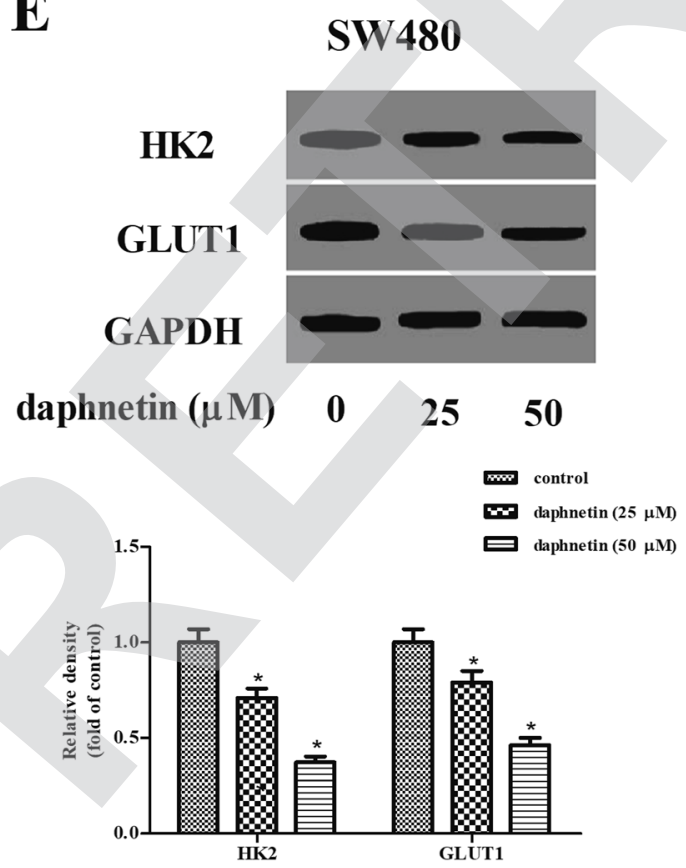

B

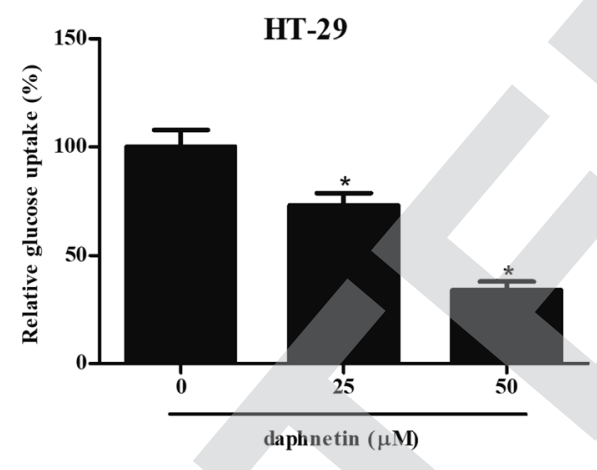

D

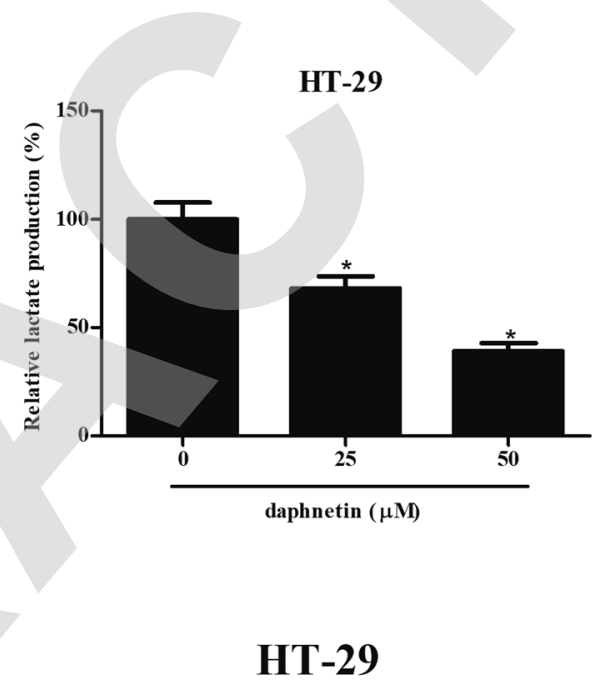

F

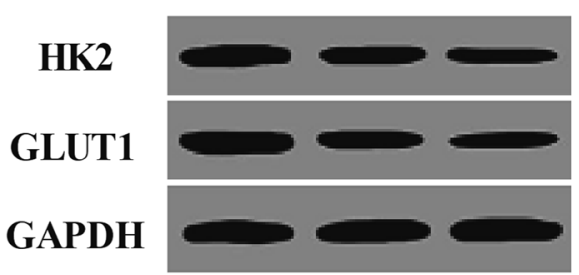

daphnetin $(\mu \mathrm{M}) \quad \mathbf{0} \quad 25 \quad \mathbf{5 0}$

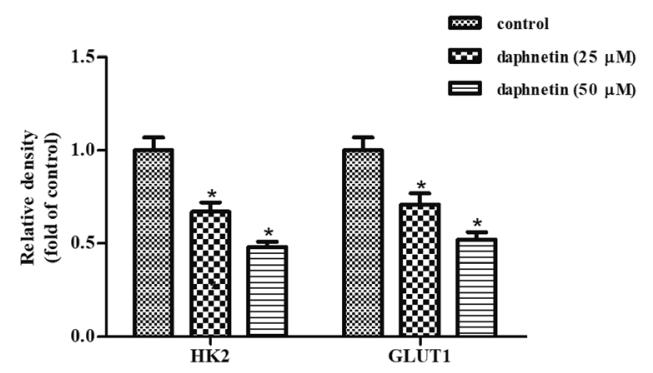

Fig. 4 Effect of daphnetin on glycolysis in CRC cells. CRC cells $\left(1 \times 10^{4}\right.$ cells per well) were treated with various daphnetin concentrations ( 0 , 25 , and $50 \mu \mathrm{M}$ ) for $24 \mathrm{~h}$. (A and B) Glucose uptake evaluated using glucose assay kit in SW480 and HT-29 cells. (C and D) Lactate level in the supernatant detected using a Lactate Colorimetric Assay Kit in SW480 and HT-29 cells. (E and F) Protein expression levels of HK2 and GLUT1 detected using western blot in SW480 and HT-29 cells. * $p<0.05$ vs. control group. 
A

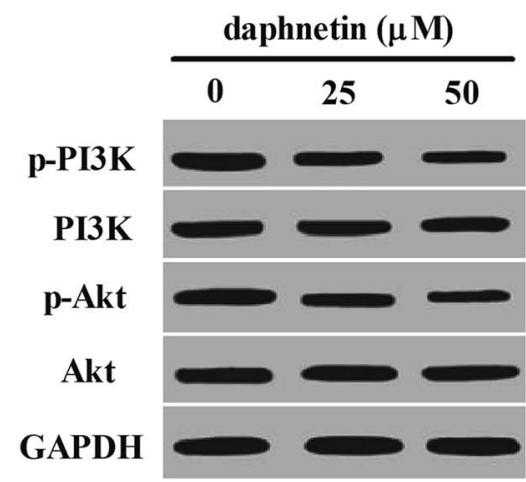

B

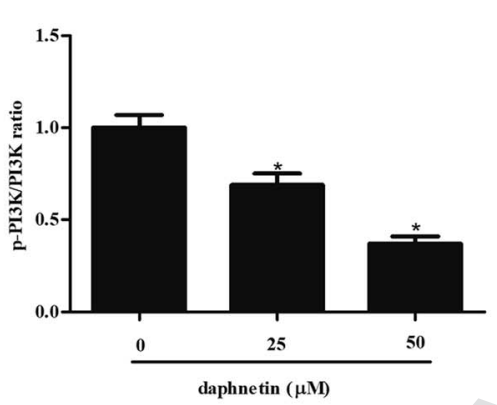

D

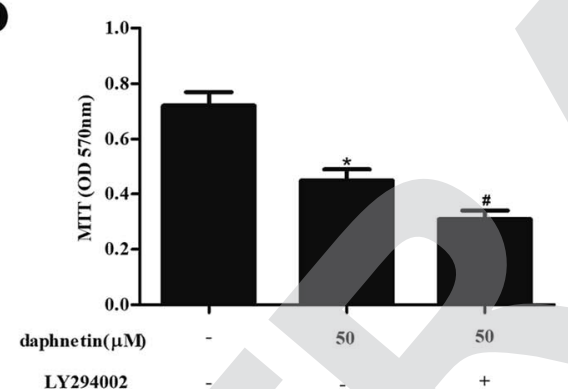

F

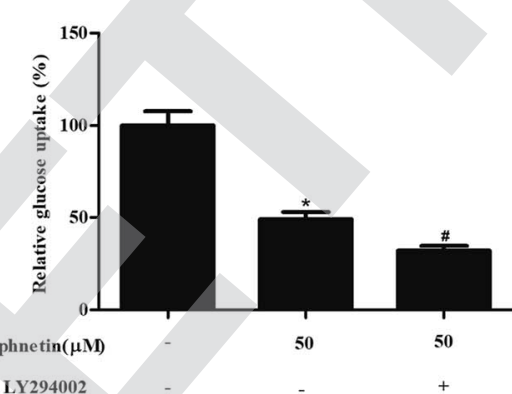

C

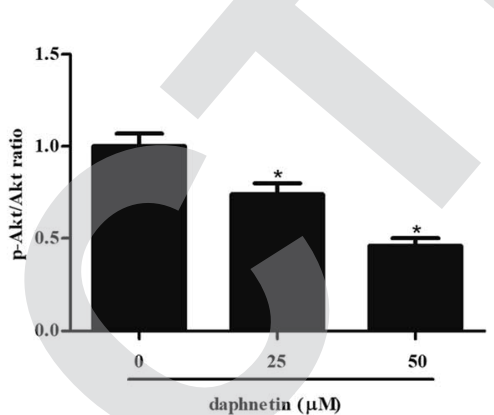

E

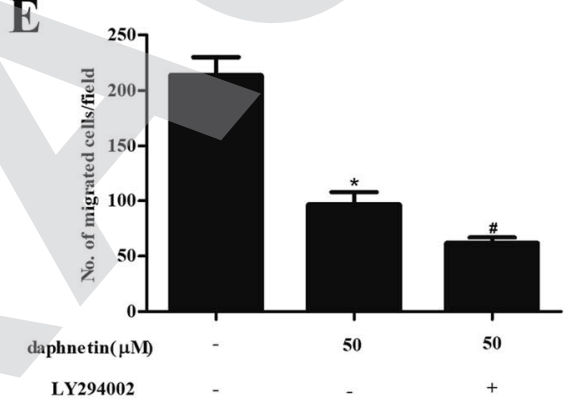

G

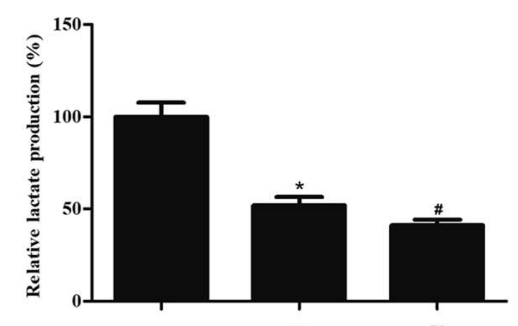

$\operatorname{daphnetin}(\mu \mathrm{M})$

LY294002
50

50

Fig. 5 Effect of daphnetin on PI3K/Akt pathway in CRC cells. SW480 cells $\left(1 \times 10^{4}\right.$ cells per well) were treated with various daphnetin concentrations $(0,25$, and $50 \mu \mathrm{M})$ for $24 \mathrm{~h}$. (A) p-PI3K, PI3K, p-Akt, and Akt expression were detected by western blot analysis. (B) Quantification analysis of p-PI3K/PI3K. (C) Quantification analysis of p-Akt/Akt. SW480 cells were pretreated with PI3K/Akt pathway inhibitor LY294002 (10 $\mu$ M) for $1 \mathrm{~h}$ and then incubated with $50 \mu \mathrm{M}$ daphnetin for $24 \mathrm{~h}$. (D) Cell proliferation determined using the MTT assay. (E) Cell migration evaluated using the transwell migration assay. (F) Glucose uptake evaluated using a glucose assay kit. (G) Lactate level in the supernatant detected using a Lactate Colorimetric Assay Kit. * $p<0.05$ vs. control group; ${ }^{*} p<0.05$ vs. daphnetin group.

PI3k/Akt signaling in SW480 cells. As shown in Fig. 5A, daphnetin treatment significantly reduced the expression of phosphorylated PI3K and Akt, while that of total PI3K and Akt remained unchanged, compared with the control group. Furthermore, pretreatment with PI3K/Akt pathway inhibitor LY294002 enhanced daphnetin-mediated cell proliferation, migration/invasion and glycolysis in SW480 cells (Fig. 5D-G).

\section{Discussion}

This study revealed for the first time that daphnetin treatment inhibited the proliferation, migration, and invasion of CRC cells, and induced CRC cell apoptosis. Furthermore, daphnetin suppressed glycolysis in CRC cells. Mechanistically, daphnetin prevented PI3K/Akt pathway activation in CRC cells. 
Daphnetin has been reported to possess antitumor activity. Fukuda et al. reported that daphnetin inhibited the invasion and migration of osteosarcoma cells. ${ }^{10}$ In accordance with previous studies, our results in this study showed that daphnetin treatment inhibited the proliferation, migration, and invasion of CRC cells. This implied that the inhibitory effect of daphnetin on CRC progression was associated with the suppression of cell proliferation and metastasis.

Most tumor cells exhibit increased glycolytic metabolism, which produces abundant ATP as the main source of energy to drive cell growth. ${ }^{12}$ Lactate, the end product of aerobic glycolysis, is often overproduced in various types of tumor and can be used to estimate the microenvironment of tumor cells. ${ }^{13}$ Our data showed that daphnetin treatment reduced lactate production in CRC cells. HK2 is an isoform of enzyme HK, which catalyzes the first step of the glycolytic pathway. ${ }^{14}$ Cancer cells express GLUT proteins at a high level. ${ }^{15}$ GLUT1 is a key glycolysis-related enzyme. The increase in glucose uptake with tumor progression was due to an increase in facilitative glucose transporters such as GLUT1. ${ }^{16}$ Our data showed that daphnetin treatment reduced the expression of GLUT1 and HK2 in CRC cells, suggesting that daphnetin might inhibit CRC cell proliferation by attenuating glycolysis-based energy metabolism.

Apoptosis, also known as programmed cell death, is an important cellular process for cell death and proliferation maintenance. ${ }^{17} \mathrm{Bcl}-2$, known as a potent inhibitor of apoptosis, inhibits mitochondrial disruption and subsequent cyt $c$ release.$^{18}$ However, bax induces a mitochondrial permeability transition by antagonizing the function of Bcl-2, resulting in cell apoptosis. ${ }^{19}$ In this study, we observed that daphnetin induced bax expression, cleaved caspase-3, and decreased bcl-2 expression in CRC cells, implying that daphnetin induces apoptosis in CRC cells by increasing the bax/Bcl-2 ratio.

Growing evidence indicates that the PI3K/Akt signaling pathway plays important roles in regulating cell proliferation, migration, and glucose metabolism. ${ }^{20-22}$ In non-neoplastic cells or normal intestinal mucosal cells, the activity of the PI3K/Akt signaling pathway is always low. Therefore, a low concentration of daphnetin might not kill non-neoplastic cells or normal intestinal mucosal cells. PI3K is activated by oncogenes, and activated PI3K can induce cancer cell proliferation and metastasis. ${ }^{23}$ Akt is a central signaling molecule in the PI3K pathway that can induce the phosphorylation and modulation of various downstream targets, including GSK-3 $\beta$ and Bad, which are implicated in cell proliferation, metastasis, and apoptosis. ${ }^{24}$ Furthermore, Akt also stimulates glucose uptake and glycolysis by upregulating the expression of glucose transporter proteins, activating glycolytic enzymes, and regulating HK expression. ${ }^{25,26}$ Therefore, targeting the PI3K/Akt pathway is a potential strategy for preventing or delaying CRC development. In this study, we found that daphnetin treatment significantly reduced the expression of phosphorylated PI3K and Akt in CRC cells. These results suggest that daphnetin inhibits CRC cell proliferation, migration, and glucose metabolism by suppressing the PI3K/ Akt signaling pathway.

In conclusion, these findings demonstrate that daphnetin inhibits cell proliferation, migration, and glucose metabolism in CRC cells. Additional studies showed that the PI3K/Akt signaling pathway is involved in the anticancer effect of daphnetin on CRC cells. Therefore, daphnetin shows potential for use as a novel anticancer agent for CRC treatment.

\section{Conflicts of interest}

The authors have no conflicts of interest.

\section{Acknowledgements}

This study was supported by the Science and Technology Foundation of Science and Technology Bureau of Henan Province, China (No. 182102310372).

\section{References}

1 R. L. Siegel, K. D. Miller, S. A. Fedewa, D. J. Ahnen, R. G. Meester, A. Barzi and A. Jemal, Ca-Cancer J. Clin., 2017, 67, 104-117.

2 S. M. Kavuri, N. Jain, F. Galimi, F. Cottino, S. M. Leto, G. Migliardi, A. C. Searleman, W. Shen, J. Monsey and L. Trusolino, Cancer Discovery, 2016, 5, 832.

3 G. Anania, G. Resta, S. Marino, N. Fabbri, L. Scagliarini, I. Marchitelli, F. Fiorica and G. Cavallesco, J. Gastrointest. Cancer, 2018, 1-11.

4 M. A. S. Chapman, J. D. Hardcastle and N. C. M. Armitage, Cancer, 2015, 76, 383-387.

5 S. Y. Lunt and M. G. Vander Heiden, Annu. Rev. Cell Dev. Biol., 2011, 27, 441-464.

6 S. O. Lim, C. W. Li, W. Xia, H. H. Lee, S. S. Chang, J. Shen, J. L. Hsu, R. Dan, D. Djukovic and H. Gu, Cancer Res., 2016, 76, 1284.

7 W. W. Yu, Z. Lu, H. Zhang, Y. H. Kang, Y. Mao, H. H. Wang, W. H. Ge and L. Y. Shi, J. Agric. Food Chem., 2014, 62, 1231512325.

8 B. Song, Z. Wang, Y. Liu, S. Xu, G. Huang, Y. Xiong, S. Zhang, L. Xu, X. Deng and S. Guan, PLoS One, 2014, 9, e96502.

9 L. Yang, Q. Yang, K. Zhang, Y. J. Li, Y. M. Wu, S. B. Liu, L. H. Zheng and M. G. Zhao, Molecules, 2014, 19, 14542.

10 H. Fukuda, S. Nakamura, Y. Chisaki, T. Takada, Y. Toda, H. Murata, K. Itoh, Y. Yano, K. Takata and E. Ashihara, Biochem. Biophys. Res. Commun., 2016, 471, 63-67.

11 L. Zhang, B. Yang and B. Yu, Biol. Pharm. Bull., 2015, 38, 1005-1011.

12 L. M. Phan, S. C. Yeung and M. H. Lee, Cancer Biol. Med., 2014, 11, 1-19.

13 H. Lu, R. A. Forbes and A. Verma, J. Biol. Chem., 2002, 277, 23111-23115.

14 H. Nilsson, D. Lindgren, A. M. Forsberg, H. Mulder, H. Axelson and M. E. Johansson, Cell Death Dis., 2015, 6, e1585.

15 C. Barron, E. Tsiani and T. Tsakiridis, BMC Proc., 2012, 6, P4. 16 P. Gonzalez-Menendez, D. Hevia, J. C. Mayo and R. M. Sainz, Int. J. Cancer, 2018, 142, 2414-2424.

17 J. F. Kerr, C. M. Winterford and B. V. Harmon, Cancer, 2015, 73, 2013-2026. 
18 J. C. Reed, J. Cell Biol., 1994, 124, 1-6.

19 K. G. Wolter, Y. T. Hsu, C. L. Smith, A. Nechushtan, X. G. Xi and R. J. Youle, J. Cell Biol., 1997, 139, 1281-1292.

20 D. Wei, F. Geng, S. Liang, H. Zhao, M. Liu and H. Wang, Biosci. Rep., 2017, 37, BSR20160470.

21 L. Yang, Y. Hou, J. Yuan, S. Tang, H. Zhang, Q. Zhu, Y. Du, M. Zhou, S. Wen and L. Xu, Oncotarget, 2015, 6, 2575525769.

22 R. Moench, T. Grimmig, V. Kannen, S. Tripathi, M. Faber, E. M. Moll, A. Chandraker, R. Lissner, C. T. Germer and A. M. Waaga-Gasser, Oncotarget, 2016, 7, 68749-68767.
23 D. A. Fruman and C. Rommel, Nat. Rev. Drug Discovery, 2014, 13, 140-156.

24 J. A. Fresno Vara, E. Casado, J. de Castro, P. Cejas, C. BeldaIniesta and M. González-Barón, Cancer Treat. Rev., 2004, 30, 193-204.

25 A. D. Kohn, S. A. Summers, M. J. Birnbaum and R. A. Roth, J. Biol. Chem., 1996, 271, 31372-31378.

26 R. L. Elstrom, D. E. Bauer, M. Buzzai, R. Karnauskas, M. H. Harris, D. R. Plas, H. Zhuang, R. M. Cinalli, A. Alavi and C. M. Rudin, Cancer Res., 2004, 64, 3892-3899. 